\title{
Necrotizing soft tissue infection on the abdominal wall. How to proceed?
}

\author{
Infección necrotizante de la pared abdominal: ¿qué hacer?
}

\author{
Raquel M. Fresnedo-Pérez ${ }^{*}$, Ana Cabrera-Pereira ${ }^{1}$, Rubén Rodríguez-García1, Desirée Díaz-González ${ }^{1}$ \\ and Javier Albaladejo-Magdalena ${ }^{2}$ \\ ${ }^{1}$ General Surgery and Digestive System Department; ${ }^{2}$ Anesthesiology and Resuscitation Department. Hospital Universitario de Cabueñes, Gijon, Spain
}

\begin{abstract}
Successful treatment of necrotizing soft tissue infections (NSTI) depends on early diagnosis and therapeutical aggressiveness, based on surgical debridement, broad spectrum antibiotics and intensive support. A case of perforated diverticulitis Hinchey 4)-secondary-NSTI is presented in order to illustrate the particular considerations in the management of abdominal wall located NSTI, supported on the evidence available in scientific literature. Several key points are identified, such as prompt and aggressive surgical debridement; culture-guided antibiotherapy and its suspension based on microbiological negativization; and the utilization of combined techniques of autoplastic repair and replacement, also supported on negative pressure therapy in the abdominal wall restoration, which have shown a good outcome.
\end{abstract}

Key words: Soft tissue infection. Abdominal wall. Necrotizing fasciitis. Acute diverticulitis.

\section{Resumen}

El éxito en el tratamiento de las infecciones necrotizantes de los tejidos blandos (INTB) depende de la precocidad diagnóstica y de la agresividad terapéutica, basada en el desbridamiento quirúrgico, la antibioticoterapia de amplio espectro y el soporte intensivo. Se presenta un caso de INTB secundaria a diverticulitis aguda perforada (Hinchey 4) con el fin de ilustrar las consideraciones particulares en el manejo de la INTB de localización en la pared abdominal, apoyadas en la evidencia disponible en la literatura científica. Se identifican como puntos clave la precocidad y la agresividad del desbridamiento quirúrgico, la antibioticoterapia dirigida por el cultivo y su suspensión guiada por la negativización microbiológica; y el empleo de técnicas combinadas de autoplastia y prótesis apoyadas en la terapia de presión negativa en la restauración de la pared abdominal, con lo que se ha obtenido un buen resultado.

Palabras clave: Infección de tejidos blandos. Pared abdominal. Fascitis necrotizante. Diverticulitis aguda.

\section{Background}

Necrotizing soft tissue infections (NSTI) are a group of diseases that affect cutaneous and subcutaneous tissues as well as superficial and deep fasciae and muscle, characterized by rapid advance of infection, which frequently evolves into a situation of sepsis or septic shock, which is why early action is required,

\section{Correspondence:}

${ }^{*}$ Raquel M. Fresnedo-Pérez 
based on aggressive surgical debridement, broad spectrum antibiotic therapy and intensive support ${ }^{1}$ in order to avoid a fatal outcome.

NSTIs can be classified according to various criteria, such as anatomical location, depth of compromised structures (adipositis, fasciitis, myositis) or causative microbial agent; or even combinations of both, such as microbial agent and depth'.

According to the microbial agent, three subtypes are described:

- Type 1 NSTIs are polymicrobial and involve aerobic pathogens (usually Streptococcus spp., Staphylococcus spp., Enterococcus spp., Enterobacteriaceae [Escherichia coli, Klebsiella spp.], Pseudomonas spp. and Acinetobacter spp.), anaerobes (Bacteroides, Clostridium spp.) and sometimes fungi. It is the most prevalent subtype, accounting for $55-75 \%$ of NSTI cases ${ }^{1}$.

- Type 2 NSTIs are monomicrobial, produced by group A streptococci, although some authors include infections caused by methicillin-resistant Staphylococcus aureus in this group?

- Type 3 NSTIs are monomicrobial, caused by Clostridium spp. or by other rare and virulent pecies, such as Vibrio vulnificus and Aeromonas spp.

Abdominal wall NSTI can originate as a complication by contiguity of an intra-abdominal process. In these cases, to infectious focus control, treatment of NSTI itself should be added, by carrying out local infection control with adequate surgical debridement and systemic treatment with life support and intravenous antibiotic therapy.

\section{Clinical case}

Seventy-year-old woman who attended the University Hospital of Cabueñes emergency department with a 5-day history of abdominal pain, vomiting and constipation. She had a medical history of morbid obesity, obstructive sleep apnea syndrome, iatrogenic hypothyroidism due to Graves-Basedow disease with thyroid ophthalmopathy, liver steatosis, chronic kidney disease of unidentified etiology and osteoporosis; as previous interventions, she had undergone hysterectomy with double adnexectomy, cholecystectomy, thyroidectomy, trabeculectomy and lumbar arthrodesis, the latter, 3 weeks before. She was on chronic treatment with thyroxine $125 \mathrm{mg}$, omeprazole $20 \mathrm{mg}$, furosemide + triamterene, prednisone $20 \mathrm{mg}$, dexketoprofen, teriparatide, tapentadol retard 50 , transdermal buprenorphine $35 \mathrm{mg}$ and clorazepate dipotassium $5 \mathrm{mg}$.

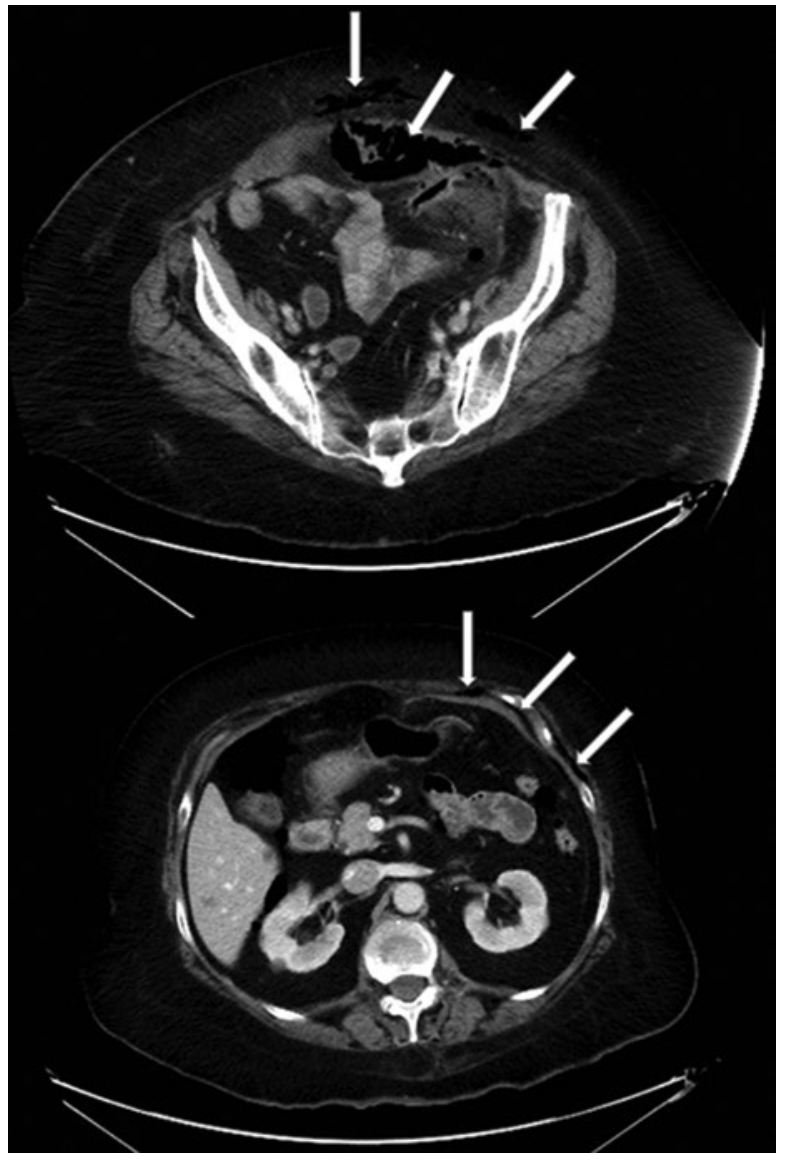

Figure 1. Computed tomography sections where the presence of gas in subcutaneous and sub-fascial planes can be appreciated.

On physical examination, a mass with phlogotic signs was detected in the infraumbilical region, highly painful to palpation The dorsal surgical wound had a good aspect.

Laboratory results showed mild leukocytosis, with 92\% neutrophilia, C-reactive protein (CPR) $206 \mathrm{mg} / \mathrm{L}$, lactate dehydrogenase $607 \mathrm{U} / \mathrm{L}$, procalcitonin $3.68 \mathrm{ng} /$ $\mathrm{mL}$, and patient's usual renal function values. Urgent abdominal computerized tomography (CT) was performed, which revealed the presence of pneumoperitoneum with small collections at the mesocolon level, thickening of adjacent fascia with multiple diverticula and free gas that extended to peritoneal fat, anterior abdominal wall muscle planes and subcutaneous tissue (Fig. 1). In view of these findings, the patient was admitted to the general surgery department to be urgently operated due to generalized fecal peritonitis secondary to perforated sigmoid diverticulitis with significant secondary infection of the abdominal wall. Hartmann sigmoidectomy with colostomy was performed in the left hypochondrium with drainage of the 
preperitoneal space, subcutaneous cell tissue and splenic space. Subsequently, she was transferred to the resuscitation unit, where paroxysmal atrial fibrillation requiring treatment with amiodarone, hypotension requiring norepinephrine administration, impaired renal function and metabolic acidosis with hyperlactatemia were recorded. Empirical antibiotic treatment at doses adjusted to her renal function was started with imipenem and vancomycin, with patient clinical and laboratory values improvement.

Worsening of sepsis parameters was observed 48 hours after the intervention in serial laboratory tests (CRP $440.2 \mathrm{mg} / \mathrm{L}$, procalcitonin $30.74 \mathrm{ng} / \mathrm{ml}$, lactate $2.2 \mathrm{U} / \mathrm{L}$ and bicarbonate $20.4 \mathrm{mEq} / \mathrm{L}$ ), and on physical examination, emission of a foul-smelling dark exudate through the abdominal wall drains was confirmed, which motivated immediate surgical reintervention. After opening the surgical wound, necrosis involving all abdominal wall planes was observed, with more remarkable involvement of the fascial plane, as well as subcutaneous adipose tissue, suggestive of necrotizing fasciitis, with associated necrosis of the muscular plane (anterior rectus and left oblique muscles) and the peritoneum itself. Necrosectomy was performed until healthy tissue was reached, including skin resection, with a loss of substance of approximately $30 \%$ of anterior abdominal wall surface; the peritoneal cavity was also examined, without pathological findings. Closure of the cavity was obviated and laparostomy coverage was carried out with Brock technique, using a polyurethane sheet as visceral protection, onto which compresses soaked in hydrogen peroxide were arranged, externally protected by a second multi-perforated polyurethane sheet. The patient was transferred to the intensive care unit.

During the first hours of the postoperative period she required hemodynamic support with norepinephrine, although spontaneous diuresis was maintained with reexacerbated renal failure values (creatinine $2 \mathrm{mg} / \mathrm{dL}$ and urea $134 \mathrm{mg} / \mathrm{dL}$ ), with vancomycin being therefore replaced by linezolid in order to avoid nephrotoxicity. A new surgical examination was carried out, where the absence of necrosis progression was observed; therefore after taking a sample for culture, a polymer film was placed for protection of the loops, and a very low-density condensate polytetrafluoroethylene mesh (OmyraMesh $^{\circledR}$ ) was fixed to prevent retraction of the aponeurosis, with new dressing on the fascial-mesh plane.

At 48 hours of the examination, another one was carried out, where the absence of infection progression was verified. The mesh was longitudinally opened at its middle part and an examination of the cavity was performed, without pathological findings. The mesh was closed with 3-4 cm approximation and the dressing was repeated after samples were taken for cultures.

Examinations were scheduled every 72 hours, placing a negative pressure system (Vacuum Assisted Closure $A B T h e r a^{\circledR}$ ) on the mesh and an intra-abdominal catheter for continuous washing with 2 liters of physiological saline every 24 hours. On day 10 surgical examination, fecal material was found in subcutaneous and intra-abdominal tissue, originating in the colostomy edge by filtration in relation to the vacuum system; therefore, not repositioning it again was decided. A thorough wash of the cavity was performed and a washing system was placed, removing the previous mesh and placing a new polypropylene and polyglecaprone mesh (PhysioMesh ${ }^{\circledR}$ ).

The first cultures reported the presence of Pseudomonas aeruginosa, Fusobacterium varium, E. coli and Enterococcus faecalis, covered with the imipenem + vancomycin/linezolid regimen. Subsequently, Candida albicans and Clostridium perfringens were detected, and anidulafungin and clindamycin were therefore added to the treatment. Each antibiotic was discontinued after negativity for the infectious agent it was aimed at was confirmed in the serial cultures.

At 32 days of the necrosectomy, sterile cultures were obtained and a control CT scan reported the absence of pathological findings. Laboratory results showed normalization of sepsis parameters and restoration of the patient baseline renal function, who was discharged from the intensive care unit and was moved to the general surgery hospitalization area.

During her stay at the hospitalization area, the negative pressure system was placed again, with development of granulation tissue, although with fascial plane dehiscence. Closure using musculocutaneous flaps was proposed, but the technique was dismissed by the plastic surgery department given the medical history of the patient; therefore, the negative pressure system was maintained until skin closure, 18 weeks after the necrosectomy. During this period, the patient received rehabilitation treatment and nutritional supplementation, and was discharged home 2 weeks after skin closure, in good general conditions, after completing a total of 20 weeks in hospitalization.

\section{Discussion}

In the available literature around NSTI, the need for an early diagnosis is specifically emphasized, 
considering that the key to a successful treatment lies in rapid decision making. We have tools available to support the NSTI diagnosis, such as the Laboratory Risk Indicator for Necrotizing Fascitis Score (LRINEC), based on CRP, leukocyte count, hemoglobin, sodium, creatinine and glucose values, which attributes a positive predictive value of $92 \%$ and a negative predictive value $96 \%$ for NSTI if a score of 6 or more points is determined. However, although there are various studies aimed at validating this diagnostic tool, their results suggest that its usefulness is greater in prognostic prediction ${ }^{1}$. On the other hand, imaging tests have a limited usefulness, since CT is only valid when gas can be detected in tissues, and magnetic resonance, although it allows to differentiate between necrotizing and non-necrotizing infections, it entails a consumption of time that is not acceptable in these cases. Thus, diagnosis must be eminently clinical and surgical ${ }^{3}$.

In the reported case, predisposing factors for the development of NSTI can be identified in patient history, such as morbid obesity, hypothyroidism, chronic kidney failure, recent surgical stress and steroid treatment, in addition to an age older than 60 years ${ }^{1}$, which, coupled with surgical findings (and with a LRINEC score of 9), are sufficient to consider the NSTI diagnosis and justifies early and aggressive surgical debridement as the first therapeutic maneuver, taking into account that the promptness and aggressiveness of necrosectomy constitute the most determining factors of expected morbidity and mortality, which is between 30 and $70 \%$ of cases, with increase being directly related to surgical delay ${ }^{4}$, as well as to patient age and presence of two or more comorbidities $^{5}$. On the other hand, the presence on CT of gas in the preperitoneal, prefascial and retrofascial and subcutaneous spaces, supports the diagnosis and makes the performance of a new test to confirm the clinical findings 48 hours after the intervention unnecessary, which would entail an unacceptable surgical delay. Moreover, this finding raises the question that perhaps broader debridement could have been indicated in the first intervention, with resection of the compromised tissue, instead of a simple drain. In the 2017 multicenter review by Faraklas et al. ${ }^{3}$, necrosectomy aggressiveness is emphasized, which must be extended until bleeding tissue is found in every case, and under no circumstances should it be closed. Examination is indicated at 24 hours in all situations to verify if there is necrosis progression, or sooner if patient clinical conditions require it. In this sense,
NSTI location in the abdominal wall should not modify the therapeutic approach, including in case of borderline tissue resection, even if it entails a loss of mutilating substance that should be restored in the future ${ }^{4}$.

There is clear evidence that NSTI first-line treatment consists of surgical debridement and broad spectrum antibiotic treatment ${ }^{3}$. However, we do not have solid recommendations about the time antibiotic therapy should be maintained. The aforementioned review by Faraklas et al. ${ }^{3}$ points out that there is no evidence that a prolonged antibiotic treatment determines better results, with septic focus control being the determining factor. In this sense, we have estimated that the most appropriate way to assess focus control is by performing serial cultures, taking samples at each surgical examination. This enables, on one hand, to modify the antibiotic treatment in order to target isolated pathogens, and on the other, to discontinue each specific drug when negative results for the pathogen it was targeted to are confirmed. This strategy allows the use of broad spectrum antibiotics to be limited and to minimize the adverse effects effects of prolonged use. In this sense, we consider an interesting option the replacement vancomycin, which is usually proposed as a treatment for gram-positive microorganisms ${ }^{1}$, by linezolid, of similar spectrum, in patients with previous kidney disease or kidney compromise during the process, in order to avoid the risk of vancomycin-induced nephrotoxicity.

Once the focus control is established, and patient stabilization achieved, we consider that obtaining sterile cultures can be a good indicator of the moment for considering abdominal wall restoration. In our case, we used a mesh with the double function of acting as an interface between the negative pressure system and the loops, and of avoiding the retraction of the aponeurotic border, thus enabling their progressive approach. Fecal contamination by aspiration from the colostomy edge limited the use of negative pressure therapy that, otherwise, as stated in the literature, is an efficacious approach in open abdomen ${ }^{6,7}$. On the other hand, closure techniques using flaps and grafts require careful and individualized assessment by the plastic surgery department, taking into account aspects of the defect itself, as well as patient conditions and medical and surgical history, which limits their application.

\section{Conclusion}

Early diagnosis, which essentially should be clinical and surgical, and rapid decision making are key to the 
success of abdominal wall NSTI treatment. The cornerstones of said treatment are aggressive surgical debridement, broad spectrum antibiotic therapy and patient intensive support, with serial surgical examinations being required in order to verify the absence of necrosis progression. Once the culture results are available, antibiotic therapy can target each infectious agent in a more specific way, adjusting it to avoid, to the extent possible, potential adverse effects.

Only when infectious focus control has been established and the patient is stable, can abdominal wall reconstruction be considered, which should be individually addressed at each case, taking into account the loss of substance, and patient status and medical history.

Rigorous observance of the recommendations in these cases can avoid a fatal outcome, even in cases of poor prognosis, as the reported one.

\section{Conflict of interests}

There are no conflicts of interest concerning the authors.

\section{Funding}

No funding has been received to carry out this work.

\section{References}

1. Cocanour CS, Chang P, Huston JM, Adams CA, Díaz JJ, Wessel CB, et al. Management and novel adjuncts of necrotizing soft tissue infections. Surg Infect. 2017;18:250-72.

2. Sarani B, Strong M, Pascual J, Schwab CW. Necrotizing fasciitis: current concepts and review of the literature. J Am Coll Surg. 2009;208:279-88.

3. Faraklas I, Yang D, Eggerstedt M, Zhai Y, Liebel P, Graves G, et al. A multi-center review of care patterns and outcomes in necrotizing soft tissue infections. Surg Infect. 2016;17:773-8.

4. Marinis A, Voultsos M, Foteinos A, Tselioti P, Avraamidou A, Paschalidis $\mathrm{N}$, et al. Necrotizing soft tissue infection of the right anterolateral abdominal wall caused by a ruptured gangrenous appendix in an elderly diabetic patient. Infez Med. 2015;23:182-6.

5. Smith NE, McKenney M. Acute sigmoid diverticulitis presenting as necrotizing fasciitis of the thigh and retroperitoneum. Am Surg. 2016; 82:301-2.

6. Tolonen M, Mentula $P$, Sallinen V, Rasilainen S, Bäcklund $M$, Leppäniemi A. Open abdomen with vacuum-assisted wound closure and mesh-mediated fascial traction in patients with complicated diffuse secondary peritonitis: a single-center 8-year experience. J Trauma Acute Care Surg. 2017;82:1100-5.

7. Acosta $S$, Bjarnason $T$, Petersson $U$, Pålsson B, Wanhainen A, Svensson M, et al. Multicentre prospective study of fascial closure rate after open abdomen with vacuum and mesh-mediated fascial traction. $\mathrm{Br}$ J Surg. 2011;98:735-43. 